\title{
A Nomogram for the Prognosis of Nasopharyngeal Carcinoma with MR Imaging-Detected Tumor Residue at the End of Intensity-Modulated Radiotherapy
}

This article was published in the following Dove Press journal:

Cancer Management and Research

Meng Xu

Chang Liu (D)

Jing Lin Mi

Ren Sheng Wang

Department of Radiation Oncology, The First Affiliated Hospital of Guangxi Medical University, Radiation Oncology Clinical Medical Research Center of Guangxi, Nanning 53002I, Guangxi, People's Republic of China
Correspondence: Ren Sheng Wang Department of Radiation Oncology, The First Affiliated Hospital of Guangxi Medical University, Radiation Oncology Clinical Medical Research Center of Guangxi, No. 6 Shuangyong Road, Nanning 53002I, Guangxi, People's Republic of China

Tel +86-77I-5359339

Fax +86-77I-535003I

Email 13807806008@I63.com
Objective: This study set out to institute an effective nomogram to predict the prognosis of nasopharyngeal carcinoma (NPC) using magnetic resonance imaging (MRI)-detected residual tumor at the end of intensity-modulated radiotherapy (IMRT).

Background: This study retrospectively analyzed the prognostic factors of NPC using MRIdetected residual tumor at the end of IMRT, in order to individualize the treatment of patients with poor prognosis as early as possible.

Methods: Overall, 162 NPC patients with local or regional residual tumor at the end of IMRT were retrospectively analyzed. Based on multivariate Cox regression analysis using the backward stepwise method, a nomogram was generated to predict the prognosis of these patients. Identification, calibration, clinical applicability and reproducibility were evaluated by $\mathrm{C}$-index, time-dependent AUC, calibration curve and bootstrap verification. According to the best cut-off value of total score of prognoses calculated by X-tile software, all patients were separated into either low-risk or high-risk group.

Results: The nomogram identified age, chemotherapy, N stage, lymph nodes necrosis are significant predictors of prognosis. The AUC of the prediction model is 0.754 , and the consistency index is 0.724 (95\% confidence interval is $0.659-0.788)$. The model has good discrimination ability. Through bootstrapping test, the consistency index, corrected slope was $0.723,0.861$, respectively. The calibration slope of predicting 3-year and 5-year overall survival was 1.006 and 1.071 , respectively. The calibration curve showed satisfactory calibration effect and good net benefit. The best cut-off value of total score of prognoses calculated by X-tile software was 149.1. Kaplan-Meier survival curve showed that OS and DMFS in the high-risk group were substantially reduced compared to those in the low-risk group.

Conclusion: We constructed and validated a new nomogram to help clinicians understand the prognosis of NPC patients with residue at the end of IMRT. With an estimate of the individual risk, clinicians can start treatment decisions as early as possible for high-risk patients with poor prognosis.

Keywords: nasopharyngeal carcinoma, residue, IMRT

\section{Introduction}

More than $70 \%$ of the new cases of nasopharyngeal carcinoma (NPC) are found in East Asia and Southeast Asia. ${ }^{1}$ The non-keratinized subtypes constitute the majority of cases in the epidemic area $(>95 \%)$, which is mainly related to EBV infection. Due to the particular anatomical position of NPC, the main treatment is 
radiotherapy. ${ }^{2}$ Over the last several years, intensitymodulated radiation therapy (IMRT) has been broadly utilized for NPC treatment. The 5-year overall survival (OS) rate is $74.6-86.3 \%$, but there still remain $10 \%$ local residual or recurrence rates. ${ }^{1}$

There are many changes in nasopharynx tissue after radiotherapy, such as edema, inflammation and fibrosis. The residual lesions detected by biopsy or imaging can be composed of either tumor stem cells, tumor cells with lost proliferative ability, and other cell types. ${ }^{3}$ For patients with residual at the end of radiotherapy, the prognosis is significantly worse. ${ }^{4,5}$ For these patients, 3 months after treatment is considered as the time point at which to evaluate the curative effect. However, according to our clinical observation, the time of tumor regression varies among patients who chose to wait, and even some patients have a time of tumor regression greater than 6 months. The biological effect of IMRT is different from that of two-dimensional radiotherapy. ${ }^{6-8}$ Also, after 3 months of observation, salvage treatment may not be effective because of the interruption of overall anti-tumor treatment. Patients with poor prognosis should be treated as early as possible to avoid treatment interruption. Therefore, we believe that there needs to be a new evaluation method to carry out risk stratification again at the end of radiotherapy in order to start the treatment of patients with poor prognosis as early as possible.

Most of the residual tumor is known to be located outside the nasopharynx. He et $\mathrm{al}^{9}$ studied 142 patients that had residual tumors after radiotherapy ended. Only $11.44 \%$ of the residual tumors were found in the nasopharynx cavity, while $30.93 \%$ were in the skull base, and $30.15 \%$ were in the parapharyngeal space and additional soft tissues. Additionally, $45.07 \%$ of patients had more than one residual tumor. In most cases, it is difficult to get a second biopsy of the tumor. Hence, clinicians have to depend on imaging to assess the effectiveness of treatment. Magnetic resonance imaging (MRI) has been considered as the recommended examination method for the diagnosis of NPC and delineation of the target area, which cannot be replaced by PET/CT scanning. ${ }^{10,11}$ It is suggested that the general accuracy of PET/CT and MRI in the diagnosis of local recurrence and residual NPC is higher, while MRI is more largely utilized in clinical practice.

To sum up, this study analyzed NPC patients with MRI-detected residual tumor at the end of IMRT and constructed an effective prognostic model to evaluate the prognosis of these patients.

\section{Materials and Methods}

\section{Patient Selection and Staging}

In this study, 162 cases of NPC from the First Affiliated Hospital of Guangxi Medical University from January 2010 to December 2012 that were initially treated with IMRT and had residual tumor were included. All patients were diagnosed using biopsy and MRI and had a complete history, physical examination, chest CT, abdominal B-ultrasound, bone scan and routine laboratory examination. The results showed no distant metastasis. At the end of radiotherapy, MRI of the nasopharynx and neck indicated local or regional residual tumor. After radiotherapy, MRI of nasopharynx and neck was followed-up. Based on AJCC/UICC 8th Edition, combined with MRI and other examinations before treatment, the location of lymph nodes was determined by RTOG (2013 Edition) neck lymph node division method. Prior to treatment, two senior radiologists evaluated the films and identified clinical manifestations of the patients, such as whether there was cranial nerve invasion or cervical lymph node palpation. This study was carried out according to the ethical standards of Helsinki Declaration and approved by the ethics committee of the First Affiliated Hospital of Guangxi Medical University. This study is a retrospective study and does not have adverse effects on the rights and health of the participants, so the requirement of informed consent is waived. At the same time, patients' privacy and personal identity information are protected.

\section{Treatment Strategy}

All 162 patients received IMRT. First, CT localization was performed, ranging from top of the skull to the lower part of the clavicular head, which had a width of $3 \mathrm{~mm}$. According to MRI, endoscopy and special conditions, the gross tumor volume of the nasopharynx (GTVnx) and gross tumor volume of the positive cervical lymph node (GTVnd) were determined. Clinical target volume-1 (CTV1) contains the whole nasopharynx mucosa and submucosa $5 \mathrm{~mm}$. The clinical target volume-2 (CTV2) contained high-risk structure on the basis of CTV1. Planning target volume (PTV) was expanded by $3-5 \mathrm{~mm}$ on the basis of each tumor target. Spinal cord, brain stem, temporal lobe, pituitary gland, optic nerve, optic chiasmata, lens, eyeball, parotid gland were protected as endangered organs. The prescription dosage was pGTVnx 68-74Gy, pGTVnd 66-70Gy, pCTV1 60-66Gy, pCTV2 50-56Gy, 5 times/week, 30-33 times in total. As per the target area and dose design guidelines of IMRT for nasopharyngeal 
Table I Characteristics of 162 Patients and Univariate Regression Analysis

\begin{tabular}{|c|c|c|c|c|}
\hline \multirow[t]{2}{*}{ Characteristics } & \multirow[t]{2}{*}{ Case Numbers (\%) } & \multicolumn{3}{|l|}{ os } \\
\hline & & $P$ value & Hazard Ratio & $95 \% \mathrm{Cl}$ \\
\hline \multicolumn{5}{|l|}{ Sex } \\
\hline Male & $132(8 \mid .5 \%)$ & Reference & - & - \\
\hline Female & $30(18.5 \%)$ & 0.345 & 0.696 & $0.328-1.477$ \\
\hline \multicolumn{5}{|l|}{ Age } \\
\hline$\geq 50$ & $57(35.2 \%)$ & Reference & - & - \\
\hline$<50$ & $105(64.8 \%)$ & 0.006 & $0.47 I$ & $0.275-0.808$ \\
\hline \multicolumn{5}{|l|}{ WHO classification } \\
\hline I & $12(7.4 \%)$ & Reference & - & - \\
\hline II & $48(29.6 \%)$ & 0.292 & 2.206 & $0.507-9.595$ \\
\hline III & $102(63.0 \%)$ & 0.234 & 2.376 & $0.57 I-9.882$ \\
\hline \multicolumn{5}{|l|}{ Chemotherapy } \\
\hline Yes & $145(89.5 \%)$ & Reference & - & - \\
\hline No & $17(10.5 \%)$ & 0.010 & 2.627 & $|.259-5.48|$ \\
\hline \multicolumn{5}{|l|}{ Treatment } \\
\hline RT alone & $17(10.5 \%)$ & Reference & - & - \\
\hline IC & $3(1.9 \%)$ & 0.585 & 0.563 & $0.07 I-4.444$ \\
\hline CCRT & $76(46.8 \%)$ & 0.04 & 0.445 & $0.206-0.963$ \\
\hline$I C+C C R T$ & $44(27.2 \%)$ & 0.026 & 0.366 & $0.15 I-0.884$ \\
\hline $\mathrm{CCRT}+\mathrm{AC}$ & $16(9.9 \%)$ & 0.77 & 0.867 & $0.334-2.250$ \\
\hline$I C+C C R T+A C$ & $6(3.7 \%)$ & 0.169 & 0.235 & $0.030-1.853$ \\
\hline \multicolumn{5}{|l|}{ Dose to GTVnx } \\
\hline$<71.2 \mathrm{~Gy}$ & $76(46.9 \%)$ & Reference & - & \\
\hline$\geq 71.2 \mathrm{~Gy}$ & $86(53.1 \%)$ & 0.434 & 1.242 & $0.722-2.139$ \\
\hline \multicolumn{5}{|l|}{ Dose to GTVnd } \\
\hline$<68.1$ Gy & $7 \mathrm{I}(43.8 \%)$ & Reference & - & - \\
\hline$\geq 68.1 \mathrm{~Gy}$ & $91(56.2 \%)$ & 0.38 & 1.28 & $0.738-2.219$ \\
\hline \multicolumn{5}{|l|}{ T stage } \\
\hline $\mathrm{TI}$ & $5(3.1 \%)$ & Reference & - & - \\
\hline $\mathrm{T} 2$ & $22(13.6 \%)$ & 0.428 & 0.438 & $0.057-3.369$ \\
\hline $\mathrm{T} 3$ & $106(65.4 \%)$ & 0.668 & 0.822 & $0.336-2.011$ \\
\hline $\mathrm{T} 4$ & $29(\mid 7.9 \%)$ & 0.274 & 0.69 & $0.356-1.340$ \\
\hline \multicolumn{5}{|l|}{$\mathrm{N}$ stage } \\
\hline No & $13(8.0 \%)$ & Reference & - & - \\
\hline $\mathrm{NI}$ & $53(32.7 \%)$ & 0.201 & 3.77 & $0.493-28.819$ \\
\hline N2 & $77(47.6 \%)$ & 0.07 & 6.3 & $0.859-46.214$ \\
\hline N3 & $19(\mid 1.7 \%)$ & 0.037 & 9.01 & I.|40-7|.207 \\
\hline \multicolumn{5}{|c|}{ Lymph nodes with fusion } \\
\hline No & $89(54.9 \%)$ & Reference & - & - \\
\hline Yes & $73(45.1 \%)$ & 0.081 & 1.62 & $0.943-2.784$ \\
\hline
\end{tabular}

(Continued) 
Table I (Continued).

\begin{tabular}{|c|c|c|c|c|}
\hline \multirow[t]{2}{*}{ Characteristics } & \multirow[t]{2}{*}{ Case Numbers (\%) } & \multicolumn{3}{|l|}{ os } \\
\hline & & $P$ value & Hazard Ratio & $95 \% \mathrm{Cl}$ \\
\hline \multicolumn{5}{|c|}{ Lymph nodes with enhancing rim } \\
\hline No & 134(82.7\%) & Reference & - & - \\
\hline Yes & $28(17.3 \%)$ & 0.077 & 1.76 & $0.94 I-3.292$ \\
\hline \multicolumn{5}{|l|}{ Lymph nodes necrosis } \\
\hline No & $110(67.9 \%)$ & Reference & - & - \\
\hline Yes & $52(32.1 \%)$ & 0.002 & 2.323 & $1.353-3.986$ \\
\hline \multicolumn{5}{|l|}{ Residual site } \\
\hline Nasopharynx residual & $83(51.2 \%)$ & Reference & - & - \\
\hline Cervical residual & $50(30.9 \%)$ & 0.567 & 1.216 & $0.622-2.370$ \\
\hline 2 sites residual & 29 (I7.9\%) & 0.421 & 1.394 & $0.620-3.132$ \\
\hline
\end{tabular}

Abbreviations: WHO, World Health Organization; GTVnx, gross tumor volume of the nasopharynx; GTVnd, gross tumor volume of the positive cervical lymph node; RT, radiotherapy; IC, induction chemotherapy; CCRT, concurrent chemoradiotherapy; AC, adjuvant chemotherapy; Cl, confidence interval; HR, hazard ratio.

carcinoma (RTOG 0225), experts agreed to set the limited dose of crisis organ: crystal $\leq 8 \mathrm{~Gy}$, parotid $\mathrm{d} 33 \leq 35 \mathrm{~Gy}$, middle ear $\leq 50 \mathrm{~Gy}$, brain stem $\leq 54 \mathrm{~Gy}$, optic nerve $\leq 54 \mathrm{~Gy}$, optic chiasmata $\leq 54 \mathrm{~Gy}$, pituitary $\leq 54 \mathrm{~Gy}$, spinal cord $\leq 45 \mathrm{~Gy}$, temporal lobe $\leq 60 \mathrm{~Gy}$, mandible and temporomandibular joint $\leq 60 \mathrm{~Gy}$, and oral radiation should be as low as possible. Eclipse radiotherapy planning system determines the planning scheme. Varian 6-MV X-ray treatment. Chemotherapy drugs were mainly platinum, and induction chemotherapy, synchronous chemotherapy or adjuvant chemotherapy were conducted according to the condition of NPC patients.

\section{Diagnostic Criteria of Local and Cervical Lymph Node Residual}

MRI diagnostic criteria of residual tumors include residual tumors within the nasopharynx or additional soft tissues after radiotherapy that acts as low signal in T1 weighted imaging, and high signal and enhancement in T2 weighted imaging. If the diameter of the short axis of the neck lymph node is greater than $10 \mathrm{~mm}$, the diameter of the retropharyngeal lymph node is greater than $5 \mathrm{~mm}$, and it still exists at the end of radiotherapy, then the local lymph node is considered residual. If the skull bone is damaged and the degree and range of bone enhancement was not decreased compared with that before radiotherapy and chemotherapy, it is considered as a residual tumor. ${ }^{4}$ The residual tumor was determined by two head and neck radiologists and two radiotherapy doctors.

\section{Follow-Up}

Follow-up methods include contacting patients or their families by telephone or according to medical records and various examinations. We checked on patients every 3 months within the first 2 years post-treatment, then every 6 months after $2-5$ years, and finally, every year thereafter. The follow-up time was from treatment completion to either time of death or final follow-up. The overall survival (OS) was measured from the day radiotherapy was completed to the day of death or final follow-up. Physical examination, microscopic examination and MRI scanning were carried out. Any clinical manifestations, CT scan or bone scan of chest and liver were recorded. Tumor response rate was determined as per the response evaluation standard of solid tumor (RECIST) version 1.0.

\section{Statistics}

Single-factor Cox regression model was used to identify the predictors, and multivariate Cox survival analysis using the backward stepwise method was carried out to identify the independent risk factors related to prognosis in the remaining patients. Additionally, the risk ratio and 95\% confidence interval (CI) correlation were calculated. The prediction model was virtualized by using a nomogram, which, in turn, was predicted by $\mathrm{C}$-index and time-dependent ROC. ${ }^{12}$ The $\mathrm{C}$-index was corrected by bootstrap verification (1000 bootstrap resampling). According to the optimal cut-off value of the total score that produced the largest $\chi^{2}$ value in the Mantel-Cox test 
Table 2 Multivariate Regression Analysis of 162 Patients

\begin{tabular}{|c|c|c|c|}
\hline Characteristics & P-value & Hazard Ratio & $95 \% \mathrm{Cl}$ \\
\hline \multicolumn{4}{|l|}{ Age } \\
\hline$\geq 50$ & Reference & - & - \\
\hline$<50$ & 0.003 & 0.435 & $0.25-0.757$ \\
\hline \multicolumn{4}{|l|}{ Chemotherapy } \\
\hline Yes & Reference & - & - \\
\hline No & 0.003 & 3.319 & $|.492-7.38|$ \\
\hline \multicolumn{4}{|l|}{$N$ stage } \\
\hline No & Reference & - & - \\
\hline $\mathrm{NI}$ & 0.138 & 4.766 & $0.604-37.583$ \\
\hline N2 & 0.051 & 7.474 & $0.992-56.3$ \\
\hline N3 & 0.025 & 11.486 & $1.358-97.123$ \\
\hline \multicolumn{4}{|c|}{ Lymph nodes necrosis } \\
\hline No & Reference & - & - \\
\hline Yes & 0.008 & 2.265 & $1.243-4.124$ \\
\hline
\end{tabular}

Abbreviations: GTVnx, gross tumor volume of the nasopharynx; $\mathrm{Cl}$, confidence interval; $H R$, hazard ratio.

assessed by X-tile software (version 3.6.1; Yale University, New Haven, CT, USA), ${ }^{13,14}$ the patients were divided into high-risk and low-risk subgroups. Kaplan-Meier survival curve was used to compare the two groups. Hazard ratio (HR), 95\% CI and log-rank p-value were recorded. SPSS 22.0 (IBM, New York, USA) and R software (version 3.5.2) with the "rms" package were used for statistics. $\mathrm{P}<0.05$ represented statistical significance.

\section{Results}

\section{Patient Characteristics and Single Factor Analysis}

Among the 162 patients in this group, 62 (38.2\%) patients achieved total remission 3 months post-treatment, and 51 (31.5\%) patients achieved total remission 3-6 months post-treatment. The 3-year and 5-year survival rates (OS) were $78.3 \%$ and $66.9 \%$, the recurrence-free survival rates (LRFS) were $91.5 \%$ and $87.3 \%$, and the distance metastasis-free survival (DMFS) were $80.0 \%$ and $73.8 \%$, respectively. Detailed baseline data are shown in Table 1. Sex, age, WHO classification, chemotherapy, treatment, dose to GTVnx, dose to GTVnd, T stage, N stage, lymph node with fusion, lymph nodes with enhancing rim, lymph nodes necrosis and residual site were analyzed by singlefactor analysis, and the results are depicted in Table 1.

\section{Multivariate Analysis and Construction of Nomogram}

In Cox multivariate regression analysis, age, chemotherapy, $\mathrm{N}$ stage and lymph node necrosis were independent prognostic markers that affect NPC patients with residual tumor. The results are summarized in Table 2. All these independent predictors were then incorporated into the nomogram. The nomogram integrated all these independent predictors, as shown in Figure 1. In the nomogram, each value of the model covariate is assigned a score of $0-100$. Through the addition of the total scores of all these predicted factors in the total subscale, we can evaluate the prognosis of NPC patients with MRIdetected residue. Scores in detail for the nomogram are shown in Table 3.

\section{Verification of Prediction Nomogram}

Each variable has a score. Through evaluating the total score of all factors on the complete subscale, the probability of certain results can be determined by making a vertical line from the complete score. The C-index of the transfer nomogram is 0.724 (95\% CI is $0.659-0.788)$. Through 1000 repeated bootstrapping test, the consistency index, corrected slope was $0.723,0.861$, respectively. Time-dependent ROC curve was utilized to verify the

Table 3 Scores in Detail for the Nomogram

\begin{tabular}{|c|c|c|}
\hline Characteristics & Assignment & Score \\
\hline \multicolumn{3}{|l|}{ Age } \\
\hline$\geq 50$ & I & 49 \\
\hline$<50$ & 2 & 0 \\
\hline \multicolumn{3}{|l|}{ Chemotherapy } \\
\hline Yes & I & 0 \\
\hline No & 2 & 66 \\
\hline \multicolumn{3}{|l|}{$\mathrm{N}$ stage } \\
\hline No & 0 & 0 \\
\hline $\mathrm{NI}$ & 1 & 33 \\
\hline $\mathrm{N} 2$ & 2 & 67 \\
\hline N3 & 3 & 100 \\
\hline \multicolumn{3}{|c|}{ Lymph nodes necrosis } \\
\hline No & I & 0 \\
\hline Yes & 2 & 49 \\
\hline
\end{tabular}

Notes: Assignment used to calculate the score. Total score $=-49 *$ Age $+66 *$ Chemotherapy $+33.4 * \mathrm{~N}$ stage $+49 *$ Lymph nodes necrosis -17.1 . 3-year Survival Probability $=2.9 \mathrm{e}-08 *$ Total score ${ }^{\wedge} 3+-3.5863 \mathrm{e}-05 *$ Total score ${ }^{\wedge} 2+0.004799804$ $*$ Total score + 0.742595659; 5-year Survival Probability $=1.18 \mathrm{e}-07 *$ Total score ^ $3+$ $-6.6583 \mathrm{e}-05 *$ Total score ${ }^{\wedge} 2+0.006322075 *$ Total score +0.732145347 . 
Points

$\begin{array}{lllllllllll}0 & 10 & 20 & 30 & 40 & 50 & 60 & 70 & 80 & 90 & 100\end{array}$

Age

$\geq 50$ years

$<50$ yesrs

Chemotherapy

no

$$
\text { yes }
$$

N stage

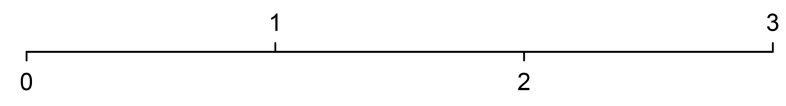

Lymph nodes necrosis

yes

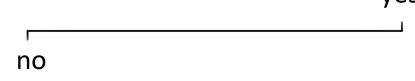

Total Points

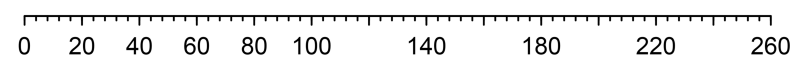

3-years Survival Probability

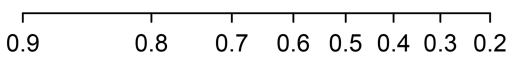

5-years Survival Probability

\begin{tabular}{llllllllll}
\hline 0.9 & 0.8 & 0.7 & 0.6 & 0.5 & 0.4 & 0.3 & 0.2 & 0.1
\end{tabular}

Figure I Nomogram for predicting the prognosis of nasopharyngeal carcinoma with MRI-detected tumor residue at the end of IMRT.

predictive nomogram, as shown in Figure 3. The AUC was

0.754, which indicates that the nomogram represents a feasible model for predicting NPC patient prognosis using an MRI-detected residual tumor. The standard curve for prognosticating patients with MRI-detected residual NPC also demonstrated suitable agreement among nomogram prediction and real-life observation, as shown in Figure 2. The calibration slope of predicting 3-year and 5 -year overall survival was $1.006,1.071$, respectively.

\section{Recognition of High- and Low-Risk} Population in Patients with Residual NPC All patients were separated to either low-risk (score $<149.1$ ) and high-risk group (score $\geq 149.1$ ). There were 141 cases in the low-risk group and 21 cases in the highrisk group. It is noted that the Kaplan-Meier survival curve showed that OS and DMFS in the high-risk group were substantially reduced compared to those in the lowrisk group $(\mathrm{P}<0.000)$, as shown in Figure 4. 

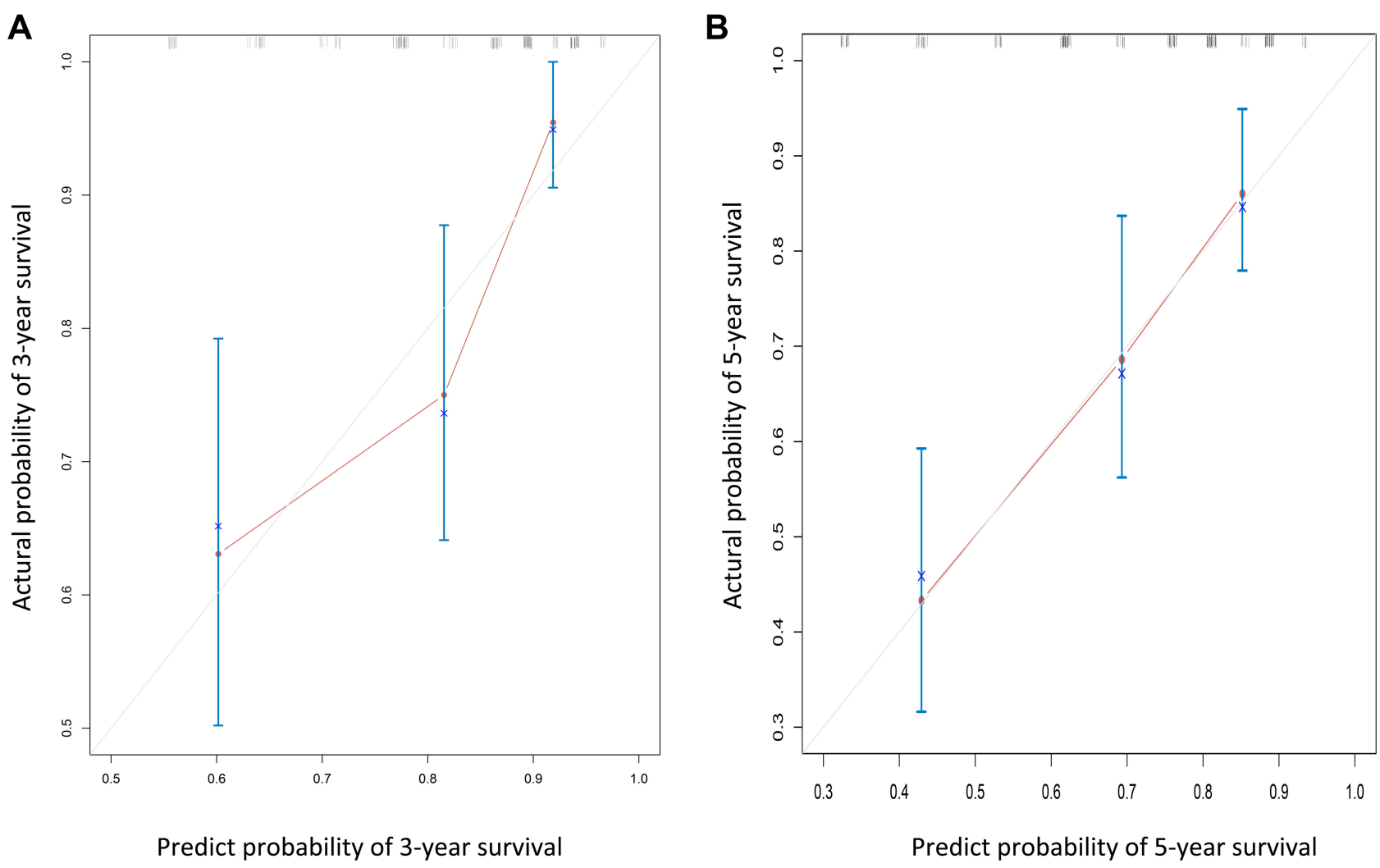

Figure 2 Calibration curves used to compare the nomogram-predicted and actual survival probabilities at 3 (A) and 5 years (B). The actual overall survival (OS) is plotted on the $y$ axis, while the nomogram-predicted probability is plotted on the $x$ axis. The dotted line indicates the reference (i.e., ideal prediction). The calibration slope of $A$ and $B$ was $1.006,1.071$, respectively.

\section{Discussion}

In the era of two-dimensional radiotherapy, a biopsy is carried out at the interval of 2 weeks after radiotherapy for NPC. Results showed that most patients achieve complete remission 11 weeks after radiotherapy. ${ }^{15}$ Therefore, it is recommended that the curative effect be evaluated 3 months post-treatment, and the patients that are identified as having residual NPC should be treated again. ${ }^{16}$ In this study, 162 patients with NPC had a local or regional residual tumor at the end of IMRT, 62 of which achieved complete remission within 3 months, and 51 of them achieved complete remission within 6 months. Some patients had complete remission for more than 6 months. Morgan et $\mathrm{al}^{17}$ used human and hamster cells to study the effect of IMRT. The results showed that IMRT prolonged the effectiveness of the same dose of irradiation, and its biological side effect (cell killing) was decreased compared to conventional and rapid irradiation. Therefore, for patients with NPC treated using IMRT, the time to complete remission may be prolonged. Liu et $\mathrm{al}^{18}$ found that the survival of patients with residual tumor 3 months after treatment was worse than that of patients without residual tumor even after remedial treatment, which may

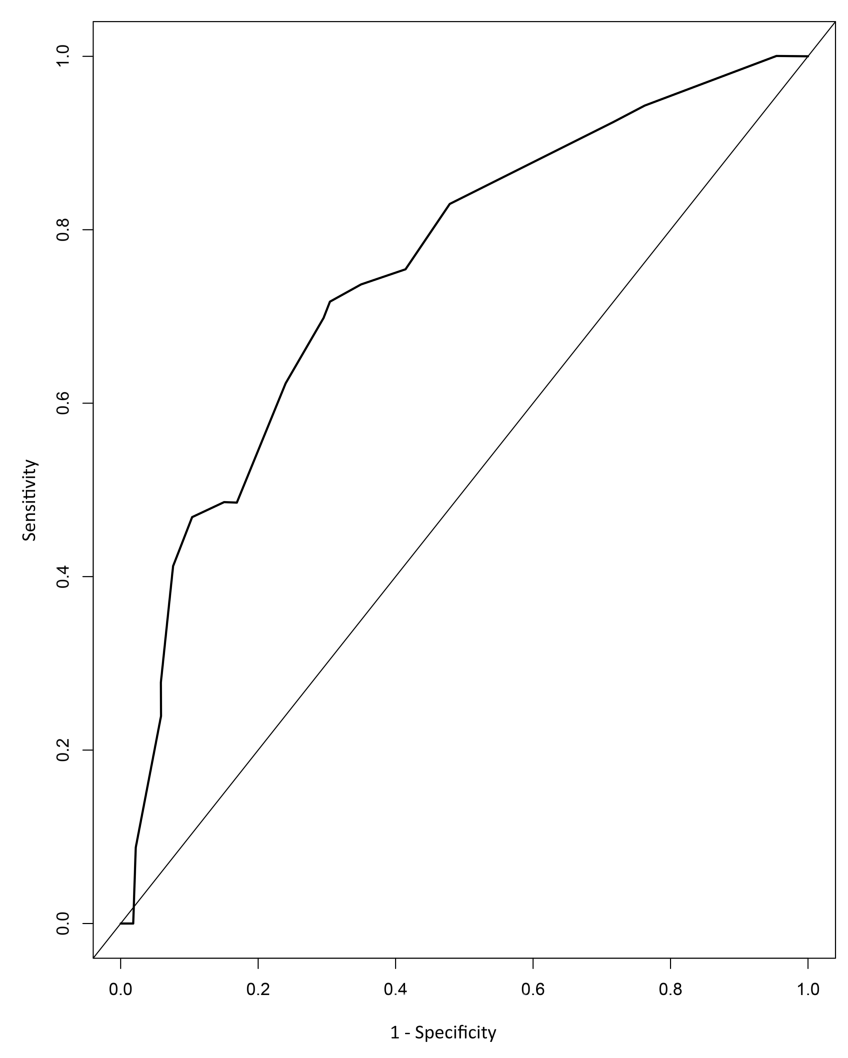

Figure 3 Operating characteristic curves (ROC) of the prediction nomogram. 

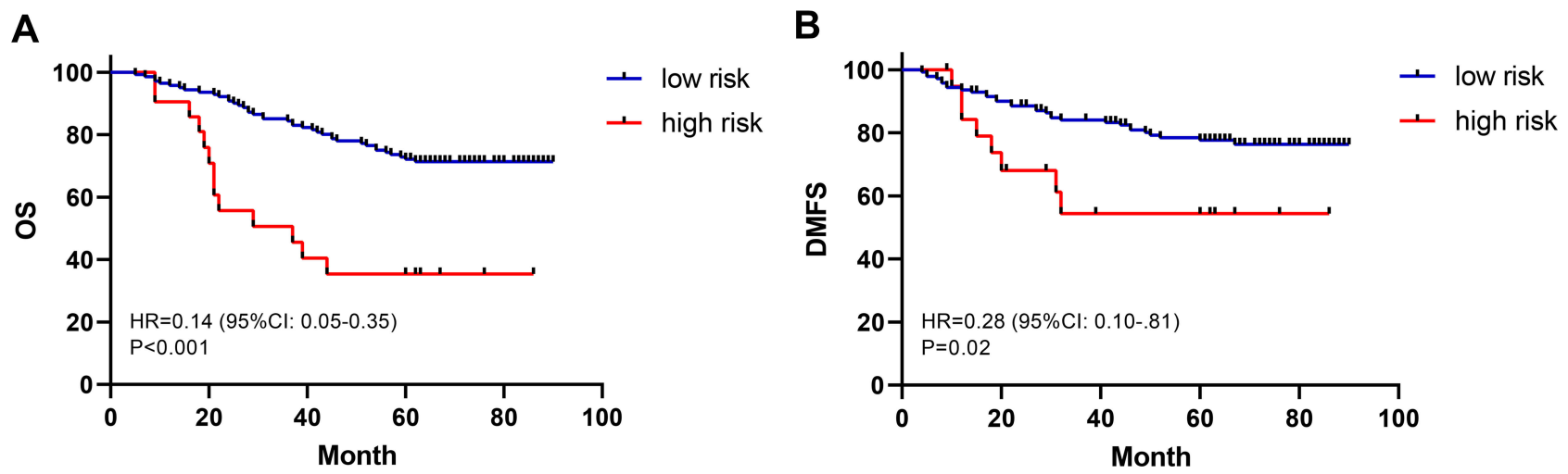

Figure 4 Overall survival curves (A) and distant metastasis-free survival curves (B) for the I62 NPC patients with residue at the end of IMRT in high-risk and low-risk groups.

be related to treatment interruption. Zhang et $\mathrm{al}^{4}$ found that image residual tumor at the end of treatment was an independent prognostic factor. Further risk stratification for these patients with poor prognosis is helpful to select high-risk patients for maintenance treatment and to avoid the effect of long-term treatment interruption. Therefore, we constructed a nomogram for the first time to predict the prognosis of patients with residual tumor at the end of IMRT, so as to start treatment as early as possible for highrisk patients, and avoid the poor effect of salvage treatment caused by an interval of 3 months.

After multivariate analysis, age emerged as an independent factor for the prognosis of OS, which is concordant with most of the current literature. ${ }^{19,20}$ Fountzilas $^{21}$ found that NPC patients younger than 50 years old had better survival prognosis than NPC patients older than or equal to 50 years old. A study of 3880 NPC patients from SEER database also confirmed age as an independent prognostic factor, as they found that the older the patients, the higher the risk of cancerrelated death. ${ }^{22}$ Poor overall condition, poor tolerance to radiotherapy and chemotherapy, and complications in elderly patients affect their prognosis.

He et al $^{9}$ suggested that $95 \%$ of $\mathrm{N}$-terminal and low GVTnx was related to the residual tumor of NPC patients. In NPC, the residual or recurrent cervical lymph nodes mostly occurred in areas II, III and V, often with large lymph node diameter or liquefied necrosis prone to residual tumor. Our findings indicate that N-stage and lymph node necrosis are also factors affecting prognosis, which may be due to the poor blood supply of the local tumor, the presence of hypoxic cells in the tumor, and insensitivity to radiation. Although the predictive value of TNM staging system is generally accepted, an increase of $\mathrm{T}$ phase and $\mathrm{N}$ Phase Is associated with a continued decline in the survival rate. ${ }^{23}$
However, T-staging was not found to affect the prognosis of patients with residual tumor after treatment ends. In these patients, there are only 4 cases of recurrence, which is far lower than the recurrence rates of most research centers. Considering that IMRT can achieve a higher dose and has superior target conformability, even in patients with late $\mathrm{T}$, local control is still effective.

In this study, patients with stage I, II and the elderly were selected as the patients for radiotherapy alone. A retrospective study was performed to evaluate the efficacy of concurrent radiotherapy and chemotherapy with that of radiotherapy alone in patients receiving IMRT. ${ }^{24}$ The results showed that OS, LRFS and DMFS of both groups were $89.8 \%$ vs $99.0 \%, 94.8 \%$ vs $89.3 \%$ and $93.4 \%$ vs $97.5 \%$ in 5 years. This study suggests that the survival benefits of concurrent radiotherapy and chemotherapy in the twodimensional era may be replaced by the survival advantages of IMRT alone, with a concurrent decrease in the incidence and degree of adverse reactions. However, there are still quite a number of clinical studies that recommend cisplatin-based chemoradiotherapy or neoadjuvant chemotherapy. Previous studies have suggested that chemotherapy can improve survival and reduce the risk of distant metastasis. ${ }^{25,26}$ In this study, multivariate regression analysis also suggested that chemotherapy could benefit in terms of long-term survival.

Our findings through multivariate Cox analysis showed that age, treatment, $\mathrm{N}$-stage, and lymph node necrosis are independent prognostic factors for residual NPC after treatment ends. The obtained nomogram shows good discrimination ability $(0.724 ; 95 \% \mathrm{CI}, 0.659-0.788)$, and the calibration curve confirms that the predicted OS probability of nomogram is in good agreement with the actual OS probability. In addition, the score generated by nomogram enables NPC patients to be additionally classified into 2 different risk 
groups. Expectedly, the proposed risk groups substantially differ with regards to the risk of OS and DMFS, particularly the high-risk group. Therefore, this nomogram may represent a clinically valuable instrument for risk stratification at the end of radiotherapy to guide the next step of treatment.

However, the nomogram validated in this study has a few limitations. Firstly, this is a retrospective study of medical archives and the sample size was small, so selection bias can occur. Secondly, the nomogram is not verified externally, but we do internal verification through 1000 bootstrap copies to evade over-fitting of results. This model is easy to use and has a relatively good accuracy. With an estimate of the individual risk, clinicians can start treatment decisions as early as possible for highrisk patients with poor prognosis.

\section{Acknowledgments}

This study was supported by the Basic Ability Enhancement Project of Young Teachers in Guangxi Zhuang Autonomous Region (No. 2018KY0134), Guangxi Science and Technology Cooperation and Exchange Project (GKH 159905-2-11), Central Guided Local Science and Technology Development Project (GK ZY18076006), Guangxi Science and Technology Program Project (GK AD17129013), and the International Communication of Guangxi Medical University Graduate Education (30/ 02103009005X).

\section{Disclosure}

The authors report no conflicts of interest in this work.

\section{References}

1. Chen YP, Chan ATC, Le QT, Blanchard P, Sun Y, Ma J. Nasopharyngeal carcinoma. Lancet. 2019;394(10192):64-80. doi:10.1016/S0140-6736(19)30956-0

2. Wei KR, Zheng RS, Zhang SW, Liang ZH, Li ZM, Chen WQ. Nasopharyngeal carcinoma incidence and mortality in China, 2013. Chin J Cancer. 2017;36(1):90. doi:10.1186/s40880-017-0257-9

3. Nicholls JM, Sham J, Chan CW, Choy D. Radiation therapy for nasopharyngeal carcinoma: histologic appearances and patterns of tumor regression. Hum Pathol. 1992;23(7):742-747. doi:10.1016/ 0046-8177(92)90342-z

4. Zhang N, Liang SB, Deng YM, et al. Primary tumor regression speed after radiotherapy and its prognostic significance in nasopharyngeal carcinoma: a retrospective study. BMC Cancer. 2014;14:136. doi:10.1186/1471-2407-14-136

5. Liang SB, Zhang N, Chen DM, et al. Prognostic value of gross tumor regression and plasma epstein barr virus DNA levels at the end of intensity-modulated radiation therapy in patients with nasopharyngeal carcinoma. Radiother Oncol. 2019;132:223-229. doi:10.1016/j. radonc.2018.10.010

6. Wu MY, He XY, Hu CS. Tumor regression and patterns of distant metastasis of T1-T2 nasopharyngeal carcinoma with intensity-modulated radiotherapy. PLoS One. 2016;11(4):e154501. doi:10.1371/journal.pone.0154501
7. Kong M, Hong SE. Tumor regression patterns by follow-up duration in patients with nasopharyngeal carcinoma treated with concurrent chemoradiotherapy. J Radiat Res. 2017;58(2):232-237. doi:10.1093/ jrr/rrw 100

8. Paganetti H. Changes in tumor cell response due to prolonged dose delivery times in fractionated radiation therapy. Int $J$ Radiat Oncol Biol Phys. 2005;63(3):892-900. doi:10.1016/j.jirobp.2005.07.953

9. He Y, Zhou Q, Shen L, et al. A retrospective study of the prognostic value of MRI-derived residual tumors at the end of intensity-modulated radiotherapy in 358 patients with locally-advanced nasopharyngeal carcinoma. Radiat Oncol. 2015;10:89. doi:10.1186/s13014-015-0401-0

10. Li Z, Li Y, Li N, Shen L. Positron emission tomography/computed tomography outperforms MRI in the diagnosis of local recurrence and residue of nasopharyngeal carcinoma: an update evidence from 44 studies. Cancer Med. 2019;8(1):67-79. doi:10.1002/cam4.1882

11. Ng SH, Chan SC, Yen TC, et al. Comprehensive imaging of residual/ recurrent nasopharyngeal carcinoma using whole-body MRI at 3 T compared with FDG-PET-CT. Eur Radiol. 2010;20(9):2229-2240. doi:10.1007/s00330-010-1784-9

12. Kamarudin AN, Cox T, Kolamunnage-Dona R. Time-dependent ROC curve analysis in medical research: current methods and applications. BMC Med Res Methodol. 2017;17(1):53. doi:10.1186/s12874-0170332-6

13. Yang K, Tian J, Zhang B, et al. A multidimensional nomogram combining overall stage, dose volume histogram parameters and radiomics to predict progression-free survival in patients with locoregionally advanced nasopharyngeal carcinoma. Oral Oncol. 2019;98:85-91. doi:10.1016/j.oraloncology.2019.09.022

14. Camp RL, Dolled-Filhart M, Rimm DL. X-tile: a new bio-informatics tool for biomarker assessment and outcome-based cut-point optimization. Clin Cancer Res. 2004;10(21):7252-7259. doi:10.1158/1078-0432.CCR-04-0713

15. Sun XS, Liu SL, Luo MJ, et al. The association between the development of radiation therapy, image technology, and chemotherapy, and the survival of patients with nasopharyngeal carcinoma: a cohort study from 1990 to 2012. Int J Radiat Oncol Biol Phys. 2019;105 (3):581-590. doi:10.1016/j.ijrobp.2019.06.2549

16. Koukourakis MI, Giatromanolaki A, Kakolyris S, et al. Phase I/II dose escalation study of docetaxel and carboplatin combination supported with amifostine and GM-CSF in patients with incomplete response following docetaxel chemo-radiotherapy: additional chemotherapy enhances regression of residual cancer. Med Oncol. 2000;17(2):135-143. doi:10.1007/bf02796209

17. Morgan GW, Breit SN. Radiation and the lung: a reevaluation of the mechanisms mediating pulmonary injury. Int $J$ Radiat Oncol Biol Phys. 1995;31(2):361-369. doi:10.1016/0360-3016(94)00477-3

18. Liu SL, Tang LQ, Chen QY, et al. The prognosis of neck residue nasopharyngeal carcinoma (NPC) patients: results from a case-cohort study. J Cancer. 2018;9(10):1765-1772. doi:10.7150/jca.24573

19. Tang LQ, Li CF, Li J, et al. Establishment and validation of prognostic nomograms for endemic nasopharyngeal carcinoma. $J$ Natl Cancer Inst. 2016;108(1):djv291. doi:10.1093/jnci/djv291

20. Li YQ, Tian YM, Tan SH, et al. Prognostic model for stratification of radioresistant nasopharynx carcinoma to curative salvage radiotherapy. J Clin Oncol. 2018;36(9):891-899. doi:10.1200/ JCO.2017.75.5165

21. Fountzilas G, Ciuleanu E, Bobos M, et al. Induction chemotherapy followed by concomitant radiotherapy and weekly cisplatin versus the same concomitant chemoradiotherapy in patients with nasopharyngeal carcinoma: a randomized Phase II study conducted by the Hellenic Cooperative Oncology Group (HeCOG) with biomarker evaluation. Ann Oncol. 2012;23(2):427-435. doi:10.1093/annonc/mdr116

22. Wu SG, Liao XL, He ZY, et al. Demographic and clinicopathological characteristics of nasopharyngeal carcinoma and survival outcomes according to age at diagnosis: a population-based analysis. Oral Oncol. 2017;73:83-87. doi:10.1016/j.oraloncology.2017.08.006 
23. Wan Y, Tian L, Zhang G, et al. The value of detailed MR imaging report of primary tumor and lymph nodes on prognostic nomograms for nasopharyngeal carcinoma after intensity-modulated radiotherapy. Radiother Oncol. 2019;131:35-44. doi:10.1016/j.radonc.2018.11.001

24. Su Z, Mao YP, Tang J, Lan XW, OuYang PY, Xie FY. Long-term outcomes of concurrent chemoradiotherapy versus radiotherapy alone in stage II nasopharyngeal carcinoma treated with IMRT: a retrospective study. Tumour Biol. 2016;37(4):4429-4438. doi:10.1007/s13277-015-4266-5
25. Liu SL, Sun XS, Yan JJ, et al. Optimal cumulative cisplatin dose in nasopharyngeal carcinoma patients based on induction chemotherapy response. Radiother Oncol. 2019;137:83-94. doi:10.1016/j. radonc.2019.04.020

26. Li WF, Chen NY, Zhang N, et al. Concurrent chemoradiotherapy with/without induction chemotherapy in locoregionally advanced nasopharyngeal carcinoma: long-term results of Phase 3 randomized controlled trial. Int J Cancer. 2019;145(1):295-305. doi:10.1002/ ijc.32099

\section{Publish your work in this journal}

Cancer Management and Research is an international, peer-reviewed open access journal focusing on cancer research and the optimal use of preventative and integrated treatment interventions to achieve improved outcomes, enhanced survival and quality of life for the cancer patient.
The manuscript management system is completely online and includes a very quick and fair peer-review system, which is all easy to use. Visit http://www.dovepress.com/testimonials.php to read real quotes from published authors. 